\title{
Erratum to: A hybrid genetic algorithm for the multi-depot open vehicle routing problem
}

\author{
Ran Liu • Zhibin Jiang • Na Geng
}

Published online: 1 October 2013

(C) Springer-Verlag Berlin Heidelberg 2013

\section{Erratum to: OR Spectrum \\ DOI 10.1007/s00291-012-0289-0}

In the published version, Table 3 contains wrong numbers which need to be corrected as follows.

For test instances R2-1 to R2-9, Cplex lower bounds (column 'Cplex $x_{\mathrm{LB}}$ ') are wrong, because Cplex computation times (column 'tc(s)') are copied to 'Cplex $\mathrm{LB}$ '. Similarly, for test instances R2-10 to R2-12, column ' $H G A_{\mathrm{b}}$ ' contains incorrect values. The correct values are shown in the following table in bold.

The online version of the original article can be found under doi:10.1007/s00291-012-0289-0.

R. Liu $\cdot$ Z. Jiang $(\varangle) \cdot$ N. Geng

Department of Industrial Engineering and Logistics Management,

School of Mechanical Engineering, Shanghai Jiao Tong University,

Shanghai 200240, China

e-mail: zbjiang@sjtu.edu.cn

R. Liu

e-mail: Liuran2009@gmail.com

N. Geng

e-mail: gengna@sjtu.edu.cn 
Table 3 Computational results on the second set random instances

\begin{tabular}{llr}
\hline Instance & $\begin{array}{l}\text { CPLEX } \\
\text { Cplex }_{\text {LB }}\end{array}$ & $\begin{array}{l}\text { HGA } \\
H G A_{\mathrm{b}}\end{array}$ \\
\hline R2-1 & $\mathbf{6 , 2 2 0 . 1 2}$ & $7,026.22$ \\
R2-2 & $\mathbf{6 , 1 6 5 . 3 0}$ & $7,263.30$ \\
R2-3 & $\mathbf{7 , 0 0 9 . 4 7}$ & $7,961.37$ \\
R2-4 & $\mathbf{6 , 6 3 1 . 3 3}$ & $7,552.12$ \\
R2-5 & $\mathbf{6 , 7 0 0 . 3 3}$ & $7,836.68$ \\
R2-6 & $\mathbf{6 , 4 3 6 . 8 8}$ & $8,078.52$ \\
R2-7 & $\mathbf{6 , 4 2 3 . 3 9}$ & $7,719.71$ \\
R2-8 & $\mathbf{7 , 2 0 1 . 8 1}$ & $8,052.16$ \\
R2-9 & $\mathbf{6 , 4 2 0 . 1 2}$ & $7,217.00$ \\
R2-10 & $7,168.17$ & $\mathbf{8 , 0 6 7 . 2 9}$ \\
R2-11 & $7,065.44$ & $\mathbf{7 , 7 1 7 . 7 6}$ \\
R2-12 & $7,551.56$ & $\mathbf{7 , 8 3 8 . 0 1}$ \\
Average & & $7,694.18$ \\
\hline
\end{tabular}

